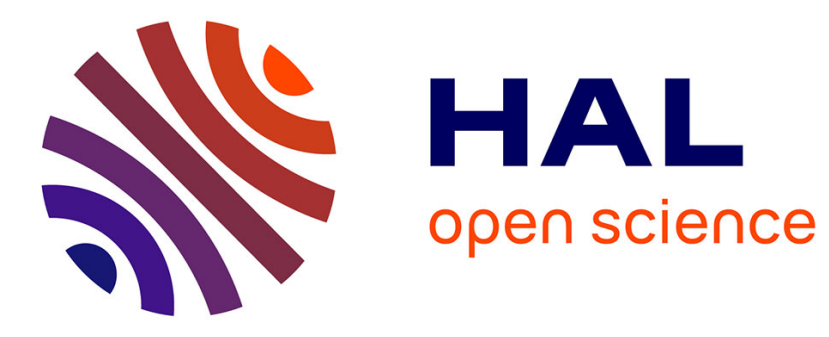

\title{
Near-field multibeam generation by tensorial metasurfaces
}

I. Iliopoulos, M. Teniou, Massimiliano Casaletti, P. Potier, P. Pouliguen, R. Sauleau, M. Ettorre

\section{- To cite this version:}

I. Iliopoulos, M. Teniou, Massimiliano Casaletti, P. Potier, P. Pouliguen, et al.. Near-field multibeam generation by tensorial metasurfaces. IEEE Transactions on Antennas and Propagation, 2019, 67 (9), pp.6068-6075. 10.1109/TAP.2019.2918490 . hal-02309548

HAL Id: hal-02309548

https://hal-univ-rennes1.archives-ouvertes.fr/hal-02309548

Submitted on 25 Nov 2019

HAL is a multi-disciplinary open access archive for the deposit and dissemination of scientific research documents, whether they are published or not. The documents may come from teaching and research institutions in France or abroad, or from public or private research centers.
L'archive ouverte pluridisciplinaire HAL, est destinée au dépôt et à la diffusion de documents scientifiques de niveau recherche, publiés ou non, émanant des établissements d'enseignement et de recherche français ou étrangers, des laboratoires publics ou privés. 


\title{
Near-Field Multi-Beam Generation by Tensorial Metasurfaces
}

\author{
Ioannis Iliopoulos, Mounir Teniou, Massimiliano Casaletti, Member, IEEE, Patrick Potier, Philippe Pouliguen, \\ Ronan Sauleau, Fellow, IEEE, and Mauro Ettorre, Senior Member, IEEE
}

\begin{abstract}
The generation of several beams with strict radiation requirements in the near field of a radiating aperture is demonstrated here. An optimization scheme is adopted to derive the aperture field distribution generating the required nearfield radiation. The optimization tool is based on a set-theoretic approach and takes into account field constraints on the radiation profile and polarization. The challenging case of generating four independent beams in the near field of a radiating aperture and with different polarizations is then considered. A tensorial metasurface is adopted to synthesize the derived aperture field profile to fully control the amplitude and phase distribution of the radiating aperture. Measurement results at $20 \mathrm{GHz}$ validate the proposed approach.
\end{abstract}

Index Terms-Aperture antennas, metasurfaces, near-field synthesis, optimization techniques

\section{INTRODUCTION}

Metasurfaces have been introduced in literature as the 2D-equivalent of metamaterials. They allow a non-canonical manipulation of the electromagnetic fields interacting with the surface [1], [2], with numerous exotic demonstrators available in the literature. Flat optics [3], perfect absorbers [4] and hologram realization [5], [6] are just a few of them. From an antenna perspective, metasurfaces provide enhanced flexibility in defining innovative radiating devices. This is clearly demonstrated by the plethora of scientific production, ranging from polarization [2], [7] and phase [8] manipulation to radiated shaped beams [9]-[14]. Metasurfaces have attracted grown interest for their capability to generate arbitrary radiation patterns at low cost and with very flat and compact structure. Nonetheless, as the antenna near field becomes more and more relevant (sensing, near-field communications, medical imaging, hyperthermia etc.) and the requirements more demanding, it is of utmost interest to combine the concept of near-field shaping and metasurfaces. The flexibility provided by the latter to mold the radiated field can significantly facilitate the realization of complex near-field distributions. However, very

This work was supported by the Centre National de la Recherche Scientifique (CNRS) and the Direction Générale de l'Armement (DGA).The authors would also like to thank Rogers Corporation for the substrate samples.

I. Iliopoulos, M. Ettorre and R. Sauleau are with the Institut d'Electronique et de Télécommunications de Rennes (IETR), UMR CNRS 6164, Université de Rennes 1, 35042 Rennes Cedex, France (e-mail: mauro.ettore@univrennes1.fr).

M. Teniou and M. Casaletti are with Sorbonne Université, Paris 06, Laboratoire d'Electronique et Electromagnétisme, L2E, F-75005, Paris, France (e-mail: massimiliano.casaletti@ sorbonne-universite.fr).

P. Potier is with the Information Superiority, DGA, Bruz 35170, France (e-mail: patrick.potier@intradef.gouv.fr).

P. Pouliguen is with Strategy Directorate, DGA, Paris 75509, France (email: philippe.pouliguen@intradef.gouv.fr). few demonstrators exist in the literature, like [11], where a Bessel aperture distribution is implemented, or [15], where a rather limiting focusing technique is used. In this work, we propose a multi-beam metasurface antenna capable of generating four beams with arbitrary polarization in close proximity to the radiating aperture. A shaping routine is introduced based on an efficient alternating projections (projections onto convex sets) optimization scheme. The shaping routine can synthesize the necessary aperture distribution, capable of radiating the aforementioned near-field profile. Specifically, it is capable of enforcing arbitrary upper bounds to the intensity (norm) of the radiated electric field, assuming a certain nearfield polarization. The synthesized aperture distribution is then practically implemented by a tensorial metasurface antenna. The latter is designed based on the methodology of [14], where a novel design methodology and class of unit cells were introduced. Thanks to this approach, vectorial aperture field distributions can be implemented using metasurfaces, used here to mold the near field.

\section{Problem Formulation And Solution}

In Fig. 1 a schematic of the problem in hand is illustrated. The target is to derive the aperture field $\boldsymbol{E}_{\mathrm{AP}}$ (electric field) able to generate a near-field distribution $\boldsymbol{E}$ with specific characteristics. In the following, we use the term "scalar" to denote the shaping/evaluation of a single field component $\left(E_{x}, E_{y}\right.$ or $E_{z}$ ) of the radiated field. Whereas, we use the term "vectorial" to refer to the manipulation of the complete electric/magnetic field vector. A fast optimization scheme capable of shaping the intensity (norm) of the electric field of continuous radiating apertures is here introduced. Despite the fact that the proposed scheme is not optimal (optimization-wise), as convex ones are [16], [17], it can mold the near field in complex shapes extremely efficiently. In the discussion to follow, normal font characters correspond to scalar quantities, bold symbols to vectors and bold symbols with a double underscore indicate dyadics. The over-line tilde corresponds to quantities in the spectral domain. The coordinate system is Cartesian, thus vectors are assumed of the form $\boldsymbol{A}=A_{x} \hat{\boldsymbol{x}}+A_{y} \hat{\boldsymbol{y}}+A_{z} \hat{\boldsymbol{z}}$, unless otherwise specified. An $e^{j \omega t}$ harmonic time dependence is assumed and suppressed.

Let us assume a circular aperture situated on the $x y$ plane of a Cartesian coordinate system and radiating in free space (permittivity $\varepsilon_{0}$, permeability $\mu_{0}$ ), as depicted in Fig. 1. The aperture field $\boldsymbol{E}^{\mathrm{AP}}$ supports only tangential-tothe-aperture components $\left(E_{z}^{\mathrm{AP}}=0\right)$ and radiates an electric 


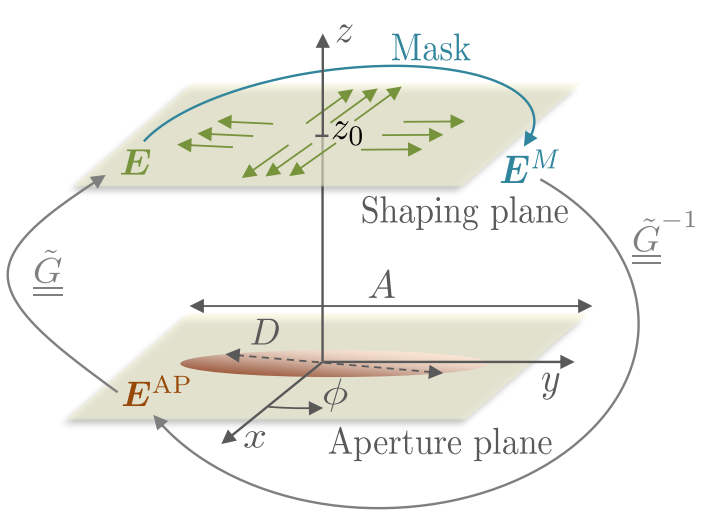

Fig. 1. Geometry of the problem.

field $\boldsymbol{E}$ in the positive half space. Our target is to find the aperture field $\boldsymbol{E}^{\mathrm{AP}}$ that generates a near field intensity $I\left(I=|\boldsymbol{E}|=\sqrt{\left|E_{x}\right|^{2}+\left|E_{y}\right|^{2}+\left|E_{z}\right|^{2}}\right)$, which is shaped according to customizable constraints at a given horizontal plane $z=z_{0}$, denoted as the "shaping plane". The proposed shaping routine is schematized in Fig. 1. It consists of three steps, which are repeated until convergence is reached ( $\boldsymbol{E}$ respects specific requirements). In particular, the optimization loop starts (step 1) with the calculation of the near field $\boldsymbol{E}$ radiated by the aperture distribution $\boldsymbol{E}^{A P}$. This is achieved by a spectral Green's dyadic $\tilde{\underline{G}}$ (see Appendix A). The initial aperture distribution is typically a uniform one, although a different choice can be made to facilitate the convergence. $\boldsymbol{E}$ is then masked (masking is detailed in the following sections) thus a slightly altered form of $\boldsymbol{E}$, namely $\boldsymbol{E}^{\mathrm{M}}$ rises (step 2). The latter is then linked to a new aperture field through the inverse dyadic $\underline{\tilde{G}}^{-1}$. Further details on the optimization scheme can be found in [18]. The masking process (step 2) of the scheme (Fig. 1) corresponds to slight alterations of the field, at the points where the latter does not obey the set requirements. Specifically, in the case under consideration, the mask is applied at the intensity of the field $I=|\boldsymbol{E}|$. It is easy to understand that the correlation of the quantity $I$ with the individual field components is not straightforward. In other words, we can construct the quantity $I$ from the individual field components but not the inverse, without any predefined relation among the components. To resolve this issue, we make two assumptions. The first one is to assume that the transverseto-the-aperture component $E_{z}$ has a much lower amplitude than the tangential ones $\left(\left|E_{z}\right| \ll\left|E_{x}\right|,\left|E_{y}\right|\right)$. This results in the following simplification

$$
I \simeq \sqrt{\left|E_{x}\right|^{2}+\left|E_{y}\right|^{2}} .
$$

The second assumption is, that, in order to maintain the phase of the shaped quantity, we define the following complex quantity

$$
I^{\prime}=\sqrt{E_{x}^{2}+E_{y}^{2}}
$$

on which the mask will be actually applied. However, the main issue of disassembling $I^{\prime}$ to the individual components on which the back-propagation can be applied is not resolved yet. The idea proposed here is to impose a specific polarization.

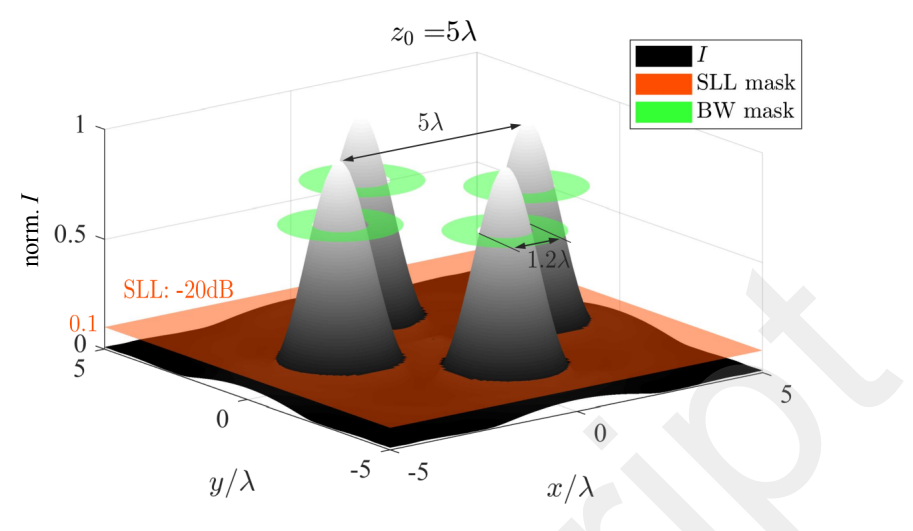

Fig. 2. Illustration of a four-beam mask.

In the following section, the application of a four-beams mask with radial polarization will be detailed.

\section{FouR-BEAMEd NEAR-FIELD INTENSITy}

The procedure detailed in section II is here applied in a multiple-beam scenario. Specifically, we search for an aperture distribution, capable of producing four beams in the near field. Additionally, we impose a radial polarization within the shaping plane $\left(z=z_{0}\right)$. The mask applied in the present case is shown in Fig. 2. The shaping plane is located at a vertical distance $z_{0}=5 \lambda$ over a circular aperture of diameter $10 \lambda$, where $\lambda$ corresponds to the free space wavelength. The applied mask (illustrated in Fig. 2) is characterized by

- Beams position: $\rho=2.5 \lambda, \phi=0: \pi / 2: 3 \pi / 2$,

- Half-power beamwidth: $1.2 \lambda$,

- Upper bounds:

- Sidelobe level (SLL): -20dB (red zone).

- Beamwidth (BW) level: -3dB (green zone)

The frequency of operation is set to $20 \mathrm{GHz}$, but the analysis and the results are inherently scalable.

The imposed radial polarization (necessary to apply the shaping routine) is here detailed. The transformation from Cartesian to cylindrical coordinates reads

$$
\begin{aligned}
& E_{x}=E_{\rho} \cos \phi-E_{\phi} \sin \phi, \\
& E_{y}=E_{\rho} \sin \phi+E_{\phi} \cos \phi .
\end{aligned}
$$

In the case of radial polarization, $E_{\phi}=0$. Let us denote as $I^{\prime \mathrm{M}}=\operatorname{Mask}\left\{I^{\prime}\right\}$ the quantity $I^{\prime}$ after the masking process has been applied. If we impose $E_{\rho}^{\mathrm{M}}=I^{\prime \mathrm{M}}$ the amplitude profile imposed by the mask can then be translated to the individual components along $x$ and $y$ as

$$
\begin{aligned}
& E_{x}^{\mathrm{M}}=E_{\rho}^{\mathrm{M}} \cos \phi=I^{\prime \mathrm{M}} \cos \phi, \\
& E_{y}^{\mathrm{M}}=E_{\rho}^{\mathrm{M}} \sin \phi=I^{\prime \mathrm{M}} \sin \phi
\end{aligned}
$$

The third step (back-propagation) becomes then feasible. The alternating projection algorithm is here outlined for clarity.

1) Evaluate the field in the region of interest

2) Mask and enforce polarization

a) Apply masking on $\left|I^{\prime}\right|=\left|\sqrt{E_{x}^{2}+E_{y}^{2}}\right|$, maintaining the phase of $I^{\prime}$ 
b) Enforce $E_{\rho}^{\mathrm{M}}=I^{\prime \mathrm{M}}$

c) Calculate $E_{x}^{\mathrm{M}}$ and $E_{y}^{\mathrm{M}}$ (equation (4))

3) Back-propagate $E_{x}^{\mathrm{M}}$ and $E_{y}^{\mathrm{M}}$ to synthesize a new aperture distribution

At each iteration, the relative error is calculated at the points where $\left|I^{\prime}\right|$ is higher than the required mask level. The error is evaluated by

$$
e=\frac{1}{N_{p}} \frac{\left|I^{\prime}(x, y)\right|-\operatorname{Mask}(x, y)}{\operatorname{Mask}(x, y)} \quad(x, y) \in \Omega_{e},
$$

where $\Omega_{e}=\left\{(x, y) \in \Omega_{\mathrm{UB}}|| I^{\prime}(x, y) \mid>\operatorname{Mask}(x, y)\right\}$ is the set where the intensity is higher than the upper bounds of the mask and $N_{p}=\mathrm{n}\left(\Omega_{e}\right)$ is the cardinality of this set. The domain $\Omega_{\mathrm{UB}}$ corresponds to the points $(x, y) \in \mathbb{R}^{2}$ where upper bounds are applied to $\left|I^{\prime}\right|$. The algorithm stops when the error remains lower than a given threshold (typically 5\%). The initial aperture distribution is generated by back-propagating the mask. At step 2, except for masking the field, it is also possible to apply symmetry constraints or to enforce a certain smoothness to the field (in the form of a Gaussian or other type of filters). The same conditions can be also applied (if necessary) to the aperture field. The procedure outlined above can be adapted to different scenarios and polarizations according to request.

\section{A. Application of the algorithm}

We may now proceed in applying the proposed algorithm in the four radially polarized beams scenario presented above. The algorithm, implemented in MATLAB, converges in 6 iterations $(<5 \%$ error) in less than $1.5 \mathrm{sec}$ on our test computer (Intel Xeon E5-2650 @ $2.6 \mathrm{GHz}, 128 \mathrm{~GB}$ RAM DDR3-1866). It should be noted that the performance is not expected to deteriorate in a lower-end computer since no parallelization was applied and the memory consumption is less than 53MB. An illustrative picture of the near field profile is depicted in Fig. 3, where the intensity $I$ is plotted for a horizontaland a vertical-to-the-aperture plane. We notice (Fig. 3a) that the beams on the $x(y)$-axis are $x(y)$-polarized, since the mask forces a radial field polarization at the shaping plane. Besides, the two beam pairs (beams along the same axis) have orthogonal polarizations. We may appreciate the appearance of the four beams at the requested plane, as well as the SLL that remains lower than $-20 \mathrm{~dB}$. The peak-to-peak distance between the beams is $5 \lambda$, as required.

It should be reminded that the target of the proposed approach is to shape the field intensity. Some assumptions were made concerning the amplitudes of the various field components. Specifically, we expect that the tangential-to-theaperture components are dominant and thus $E_{z}$ is sufficiently low. This is indeed valid, as illustrated in Fig. 4. Not only we notice that $E_{\rho}$ is dominant, but also that the mask is very well respected. Besides, the intensity of the field respects the requirements and the $E_{z}$ component plays a minimal role to the intensity (except for a small region at the center).

By also observing Fig. 5, the same conclusions can be reached. Specifically, a comparison between $\left|E_{\rho}\right|$, the intensity of the tangential field $\left(E_{\mathrm{t}}=\sqrt{\left|E_{x}\right|^{2}+\left|E_{y}\right|^{2}}=\right.$

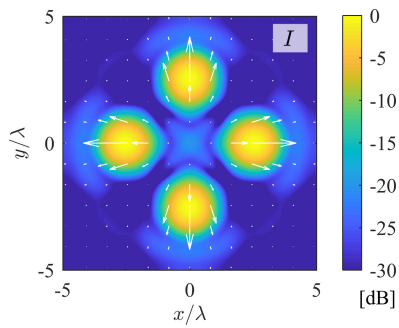

(a)

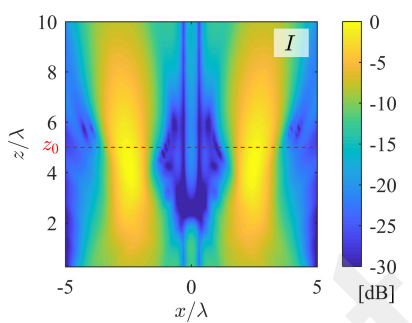

(b)
Fig. 3. 2-D illustrations of the normalized field intensity $I$ in $\mathrm{dB}$ at (a) the plane $z=z_{0}=5 \lambda$, (b) the $x z$-plane. The $y z$-plane is identical and is omitted for brevity. The white arrows correspond to the field polarization.

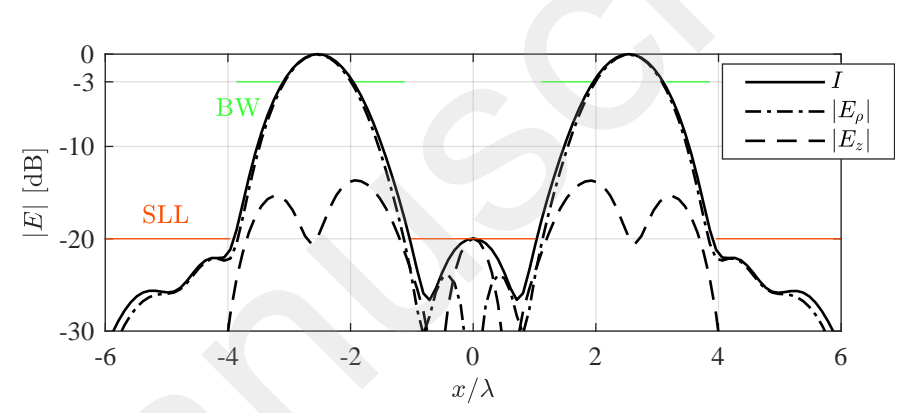

Fig. 4. Comparison of the field components along $x$-axis on the shaping plane. The components are normalized to the field intensity $I$.

$\sqrt{\left|E_{\rho}\right|^{2}+\left|E_{\phi}\right|^{2}}$ ) and the total field intensity $I$ is illustrated. We may, therefore, conclude that the shaping goals have been met since the intensity of the field has been successfully shaped. Additionally, we may also notice the successful enforcement of the requested polarization, since the $E_{\phi}$ component (Fig. 5b) is less than $-30 \mathrm{~dB}$ along the whole shaping plane. The limitation of this technique stems from the assumption concerning the low power carried by the $E_{z}$ component. This assumption might not hold for distances very close to the aperture (less than the aperture radius). In fact, $E_{z}$ can be dominant in this region and possibly different assumptions should be made in the first place. Nevertheless, the shaping distance for this example is half aperture diameter, showing that this shaping scheme can be applied to rather close to the antenna distances, rendering the technique sufficiently versatile. Up to this point, only the resulting near field has been investigated. It has been shown that the shaping process was successful since the resulting field intensity obeys the provided mask. However, the aperture field that generates this near field is of utmost importance and will be now discussed. The field over the aperture plane is plotted in Fig. 6. Despite the fact that the near field at the shaping plane is strongly radially polarized $\left(\left|E_{\phi}\right| \ll\left|E_{\rho}\right|\right.$, Fig. 5b), the aperture field appears to have regions where the $\phi$-component affects the vector orientation. This is clearly illustrated in Fig. 6, where we notice that $\max \left\{\left|E_{\phi}^{\mathrm{AP}}\right|\right\} \simeq 0.7 \max \left\{\left|E_{\rho}^{\mathrm{AP}}\right|\right\}$, with $\max$ the maximum operator. This shows that despite the fact that the near-field distribution in radially polarized, the aperture field necessary to radiate it has an important $\phi$ component. 


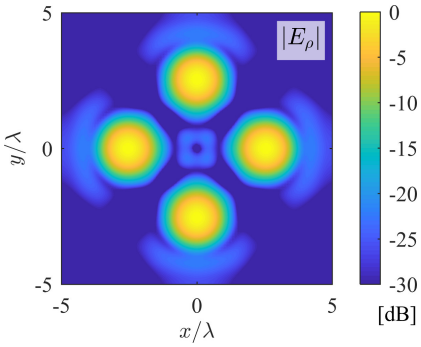

(a)

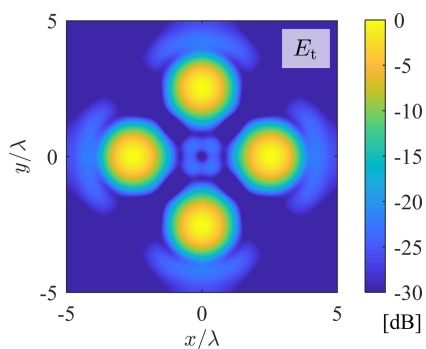

(c)

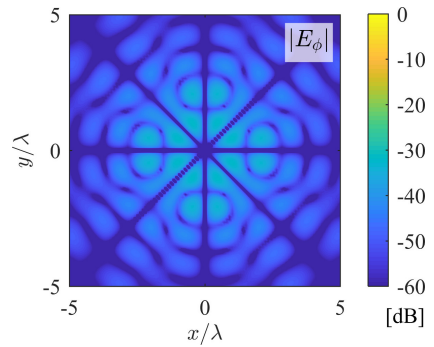

(b)

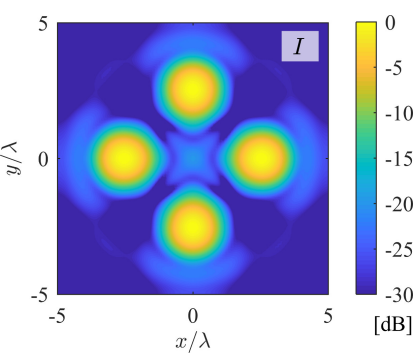

(d)
Fig. 5. 2-D illustrations at the shaping plane $\left(z=z_{0}=5 \lambda\right)$ in $\mathrm{dB}$ of (a) $\left|E_{\rho}\right|$, (b) $\left|E_{\phi}\right|$, (c) the tangential field $E_{\mathrm{t}}$ and (d) the intensity of the field $I$. We notice that the amplitude of $E_{\rho}$ is almost equal to $I$. The plots are normalized to the total field intensity $I$.

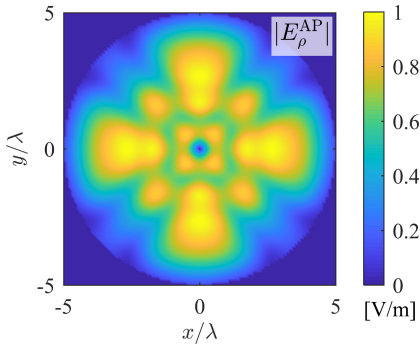

(a)

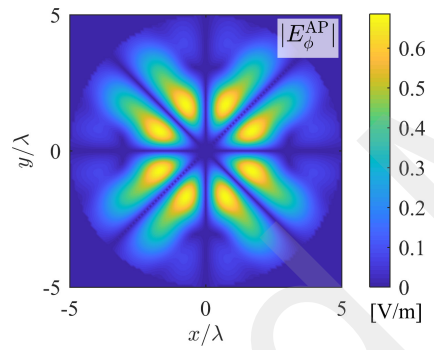

(b)
Fig. 6. Comparison between the (a) $\rho$ and (b) $\phi$ component of the aperture distribution. $\left|E_{\phi}^{\mathrm{AP}}\right|$ is normalized to the maximum of $\left|E_{\rho}^{\mathrm{AP}}\right|$.

\section{REALIZATION OF THE APERTURE FIELD}

We now consider the practical implementation of the derived aperture distribution $\left(\boldsymbol{E}^{\mathrm{AP}}\right)$. Metasurfaces are here adopted to approximate in the best possible way a continuous aperture. Such a solution also offers the freedom of controlling not only the field orientation and phase over the aperture, but also the amplitude [14], [19].

\section{A. Metasurface Design}

The metasurface is designed based on the local holography and variable impedance modulation procedure detailed in [14]. The unit cell consists of a V-shaped cut out of a circular patch depicted in Fig. 7. The periodicity (unit cell lateral dimension) is $d^{\prime}=\lambda / 12$. The geometrical parameters $\theta, g$ and $\psi$ (corresponding to the angle, width, and orientation of the cut, respectively) control the tensorial surface impedance of the unit cell. A detailed explanation of the complete design process and the unit cell properties have been presented in [14] and will be avoided here for brevity.

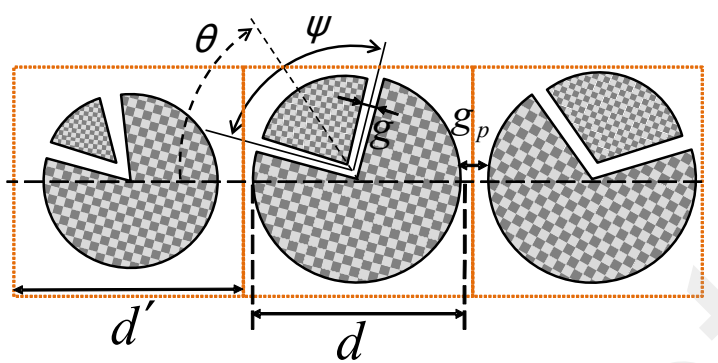

Fig. 7. Unit cells ( $d$ is the patch dimension, $d^{\prime}$ is the spatial periodicity of the lattice, $\theta$ is the patch rotation angle). The slot is described by the opening angle $\psi$ and its width $g$.

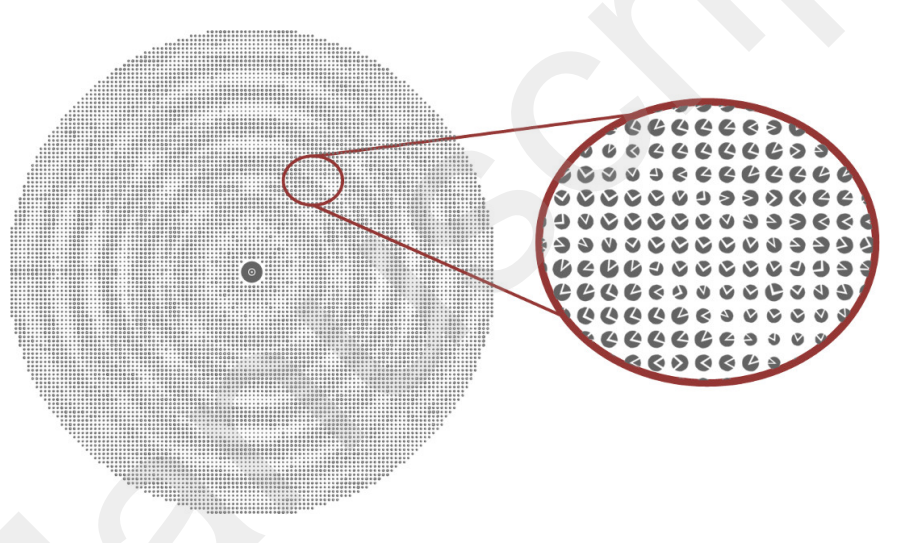

Fig. 8. Top view of the designed metasurface.

The resulting metasurface is illustrated in Fig. 8. It consists of a total of 10732 unit cells (double this number for the elements) and its diameter is $150 \mathrm{~mm}$ (or $10 \lambda$ at $20 \mathrm{GHz}$ ). The metasurface is supported by a Rogers TMM6 substrate of $1.27 \mathrm{~mm}$ thickness. The central disk and the surrounding annulus, serve for matching the antenna to the input coaxial connector ( $\Sigma$ RI 25-130-1000-90, $2.92 \mathrm{~mm}$ ).

In order to simulate the structure, we used the HFSS 3D Layout of the ANSYS Electronics Desktop Suite.The radiated near field of the antenna is evaluated form the current distribution provided by the full-wave simulator.

In Fig. 9 a comparison with the near field generated by the theoretical continuous aperture is presented. The main beams are reproduced by the metasurface accurately, but we observe the appearance of higher sidelobes. While the sidelobes on the external sides of the beams are relatively close to the $-20 \mathrm{~dB}$ requirement imposed in the theoretical work, at the center, they almost reach $-10 \mathrm{~dB}$. The latter can be associated with the feeding mechanism (which was not taken into account in the theoretical calculations) [20].

A closer investigation has been performed by modeling our antenna using a 2.5d Method of Moments algorithms. This latter, using an infinite dielectric Green's function cannot model the edge diffraction of the surface wave, as a result, the artifacts present in the radiation pattern are essentially due to the feeder. Fig.10 presents the near-field obtained with the commercial software ANSYS Designer. In Fig.10a a horizontal cut at a height $z=5 \lambda$ is represented, while Fig.10b shows the vertical cut-planes of the near field. It can be seen a higher level of the field amplitude close to the metasurface, 


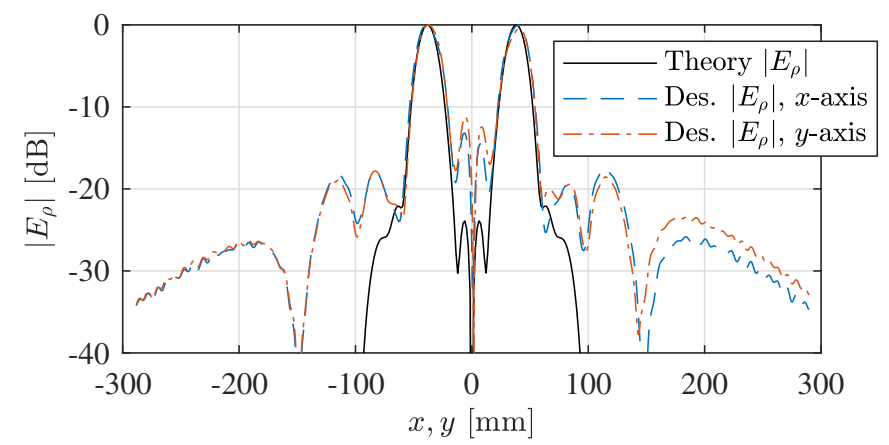

Fig. 9. Comparison between HFSS 3D Layout (denoted "Des.", as of Designer) and the near field generated by the continuous aperture distribution along the $x$ and $y$ axis.

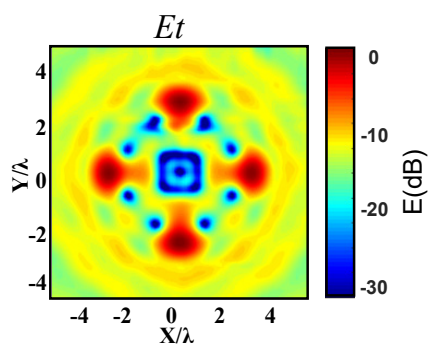

(a)
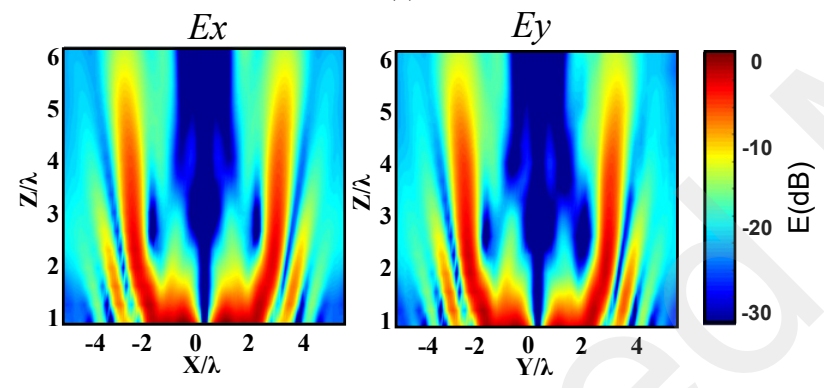

(b)

Fig. 10. 2.5d MoM radiated near-field. (a) Horizontal cut-plane and (b) Vertical cut-plane.

especially at its center (feeder position).

As second step, a non-modulated metasurface excited by the same feeder is modeled. As the surface impedance is constant, only a surface wave component is supported by the metasurface. As a consequence, the field above the metasurface is mainly due to the feeder. The idea is to subtract this nearfield from the antenna near-field patterns. Even though this approach is not exact, it can give an idea about the spurious effects of the feeder.

Fig.11 shows the results of this procedure applied to the horizontal and vertical cut-planes. We can conclude that the metasurface radiates a near-field pattern very close to the ideal one, while the feeder significantly affects the near field, leading to higher sidelobes.

In general, we acknowledge an overall satisfactory agreement (taking into account the complexity of the aperture distribution).

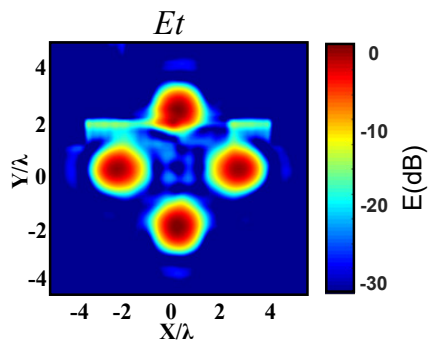

(a)
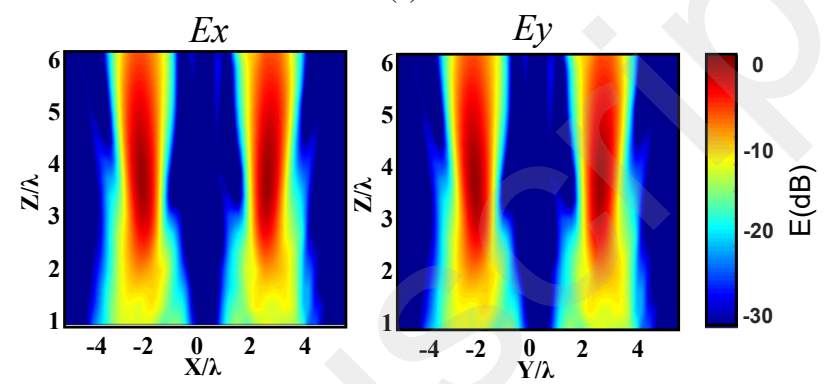

(b)

Fig. 11. 2.5d MoM radiated near-field without the feeder contribution. (a) Horizontal cut-plane and (b) Vertical cut-plane.

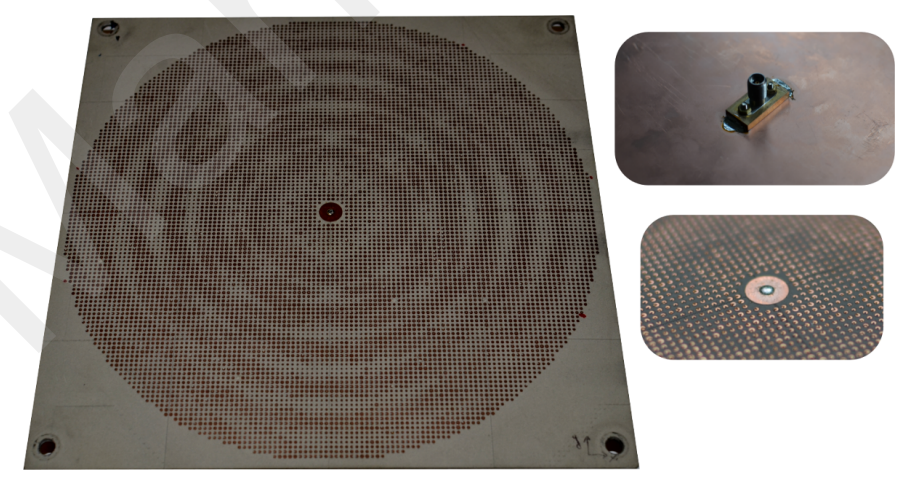

Fig. 12. Picture of the manufactured prototype. Visible in the insets are the connector on the back side of the antenna and its connection at the top surface.

\section{B. Experimental Validation}

In order to experimentally validate the shaping process and the metasurface design, a prototype was manufactured and measured. The prototype was fabricated using an LPKF ProtoLaser S laser system. The final prototype is depicted in Fig. 12.

The measurements of the prototype took place in the near-field test range of IETR. The measurements set-up is illustrated in Fig. 13. The used near-field probe is a WR42 probe from Flann Microwave. The flange of the probe is parallel to the metasurface plane, as a result, we measure only tangential-to-the-aperture components. A standard probecorrection technique for planar near-field measurements [21], [22] was applied in order to remove the effect of the measuring probe. The measurement step in all directions was set to $2 \mathrm{~mm}$ or $\lambda / 7.5$ at $20 \mathrm{GHz}$.

The measured near field at the shaping plane $\left(z_{0}=75 \mathrm{~mm}\right)$ is presented in Fig. 14 in terms of the $E_{\rho}$ component. We observe the four beams, two per polarization. The relatively "noisy" is due to spurious radiation originating from edge 


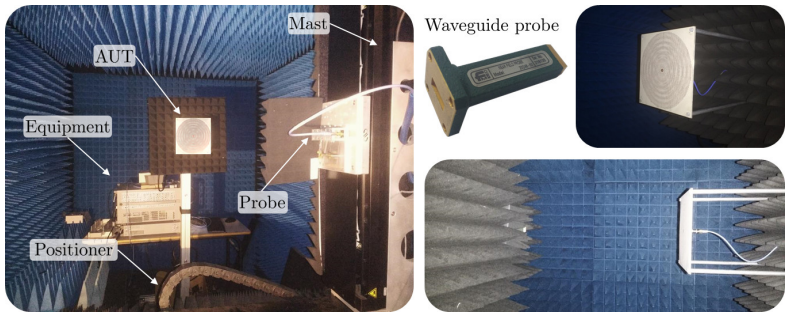

Fig. 13. Details of the measurement set-up.

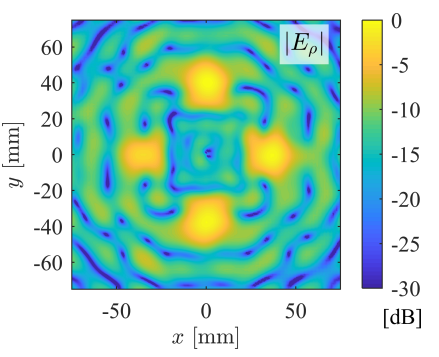

(a)

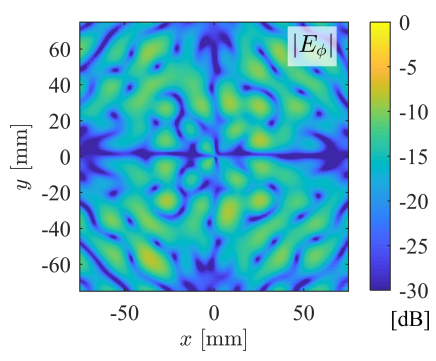

(b)
Fig. 14. Illustrations of the measured $(20 \mathrm{GHz})$ near-field at the shaping plane $z_{0}=5 \lambda=75 \mathrm{~mm}$. 2-D plots of the normalized amplitude of (a) $E_{\rho}$ and (b) $E_{\phi}$.

effects. This results also to some oscillations in the measured reflection coefficient depicted in Fig. 15. Nevertheless, the agreement between measurement and simulation of the $S_{11}$ is quite satisfactory.

A comparison between simulation and measurement is depicted in Fig. 16. A good agreement can be appreciated when it comes to the locations of the beams and the sidelobes close to zero for both axes. Especially along the $y$-axis, the agreement is very satisfactory. However, a certain disagreement is present when it comes to the outer sidelobes. Specifically, we observe sidelobes around $-8 \mathrm{~dB}$ and $-12 \mathrm{~dB}$ along the $x$ - (black) and $y$ - (red) axis, respectively. This is the result of the truncation of the antenna, which cannot be taken into account in the simulations.

For completeness, the field on the two main vertical planes, namely $x z$ and $y z$ is illustrated in Fig. 17. We observe that the field on the $x z$ plane is slightly asymmetric.On the contrary, the shape of along the $y z$ plane is very satisfactory.

An estimation of the antenna efficiency has been obtained by integrating the z-component of the Poynting vector. The

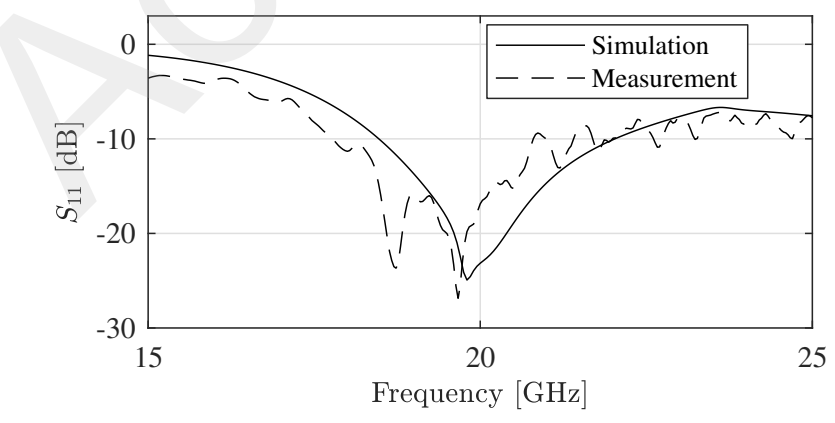

Fig. 15. Measured and simulated (HFSS) reflection coefficient.

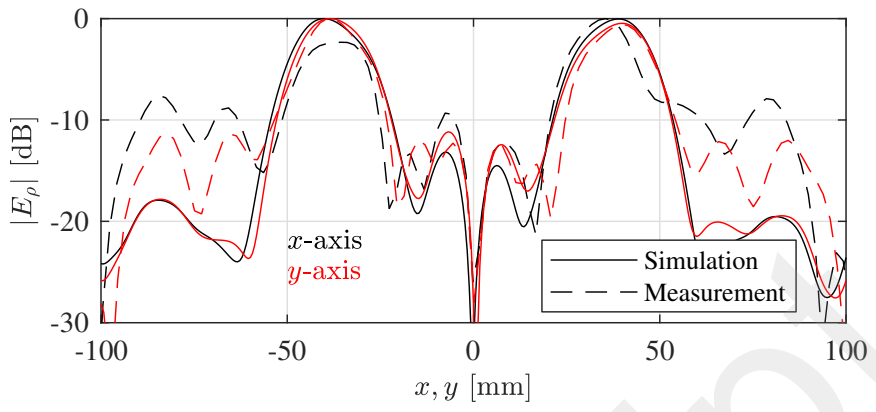

Fig. 16. Measured and simulated (HFSS 3D Layout) near field at $20 \mathrm{GHz}$ on the shaping plane $z_{0}=5 \lambda=75 \mathrm{~mm}$ along the $x$ (black) and $y$ (red) axis.

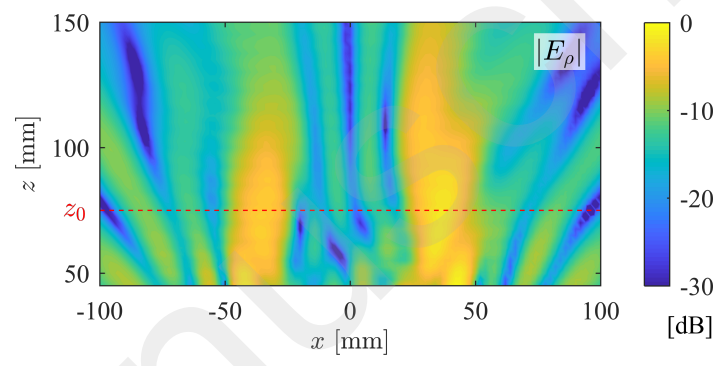

(a)

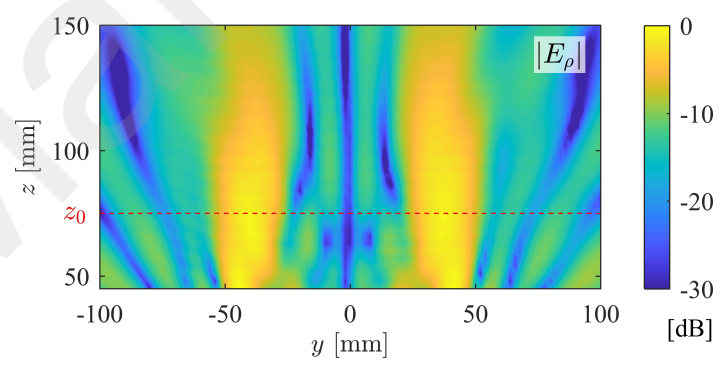

(b)

Fig. 17. Amplitude of the measured $\left|E_{\rho}\right|$ component on the (a) $x z$-plane and (b) $y z$-plane. The red line corresponds to the shaping plane $z=z_{0}$.

radiated power $\left(P_{r a d}\right)$ is obtained by performing the integral at the aperture plane (metasurface currents), while the power associated with the beams $\left(P_{\text {beams }}\right)$ is calculated at the shaping plane $(z=75 \mathrm{~mm})$ integrating within the area defined by the focal spot of the four beams. The radius of each circular spot has been defined as for the Gaussian beams case, namely $w(z)=\frac{F W H M(z)}{\sqrt{2 \ln 2}}=0.875 \lambda$. The ratio $P_{\text {beams }} / P_{\text {rad }}=68.56 \%$ gives us an estimate of the power confinement efficiency of the metasurface (in the ideal case of 4 Gaussian Beams the ratio $P_{\text {beams }} / P_{\text {rad }}=86.5 \%$ ).

\section{CONCLUSION}

The capability to shape the intensity of the near field generated by a tensorial metasurface on 2-D planes has been demonstrated. The proposed technique involves an alternating projection method which can apply a requested mask on the field intensity, subject to a specified field polarization. To demonstrate the efficacy of the algorithm, a near-field distribution presenting four radially polarized beams at a vertical distance of half an aperture diameter was successfully engineered. The beams present a beamwidth of $1.2 \lambda=18 \mathrm{~mm}$ 
and are separated by a horizontal distance of $5 \lambda=75 \mathrm{~mm}$. The achieved SLL is $-20 \mathrm{~dB}$. Despite the choice for this specific distribution, different field masks can be used.

The theoretical results have also been experimentally validated. Specifically, an anisotropic metasurface was designed, fabricated and measured. The measured fields show a good agreement with the predictions, thus validating the overall design process.

\section{APPENDIX A}

\section{Evaluation of the NeAR Field}

Let us assume a circular aperture situated on the $x y$ plane of a Cartesian coordinate system and radiating in free space (permittivity $\varepsilon_{0}$, permeability $\mu_{0}$ ), as depicted in Fig. 1. The aperture field $\boldsymbol{E}^{\mathrm{AP}}$ supports only tangential-tothe-aperture components $\left(E_{z}^{\mathrm{AP}}=0\right)$ and radiates an electric field $\boldsymbol{E}$ in the positive half space. Our target is to find the aperture field $\boldsymbol{E}^{\mathrm{AP}}$ that generates a near field intensity $I\left(I=|\boldsymbol{E}|=\sqrt{\left|E_{x}\right|^{2}+\left|E_{y}\right|^{2}+\left|E_{z}\right|^{2}}\right)$, which is shaped according to customizable constraints at a given horizontal plane $z=z_{0}$, denoted as the "shaping plane".

In order to accelerate the evaluation of the near field we adopt a spectral approach, where the spectral representations of the aperture $\left(\tilde{\boldsymbol{E}}^{\mathrm{AP}}\right)$ and radiated near field $(\tilde{\boldsymbol{E}})$ are linked by a spectral dyadic Green's function $\underline{\tilde{G}}$ as

$$
\tilde{\boldsymbol{E}}=\underline{\underline{\tilde{G}}} \cdot \tilde{\boldsymbol{E}}^{\mathrm{AP}} .
$$

The Green's dyadic is given by [23, sec. 12.9]

$$
\begin{aligned}
\underline{\underline{\tilde{G}}}(z) & =e^{-j k_{z} z} \hat{\boldsymbol{x}} \hat{\boldsymbol{x}}+e^{-j k_{z} z} \hat{\boldsymbol{y}} \hat{\boldsymbol{y}} \\
& -\frac{k_{x}}{k_{z}} e^{-j k_{z} z} \hat{\boldsymbol{z}} \hat{\boldsymbol{x}}-\frac{k_{y}}{k_{z}} e^{-j k_{z} z} \hat{\boldsymbol{z}} \hat{\boldsymbol{y}},
\end{aligned}
$$

where $k_{x}, k_{y}$ and $k_{z}=\sqrt{k^{2}-k_{x}^{2}-k_{y}^{2}}\left(-\pi<\arg \left\{k_{z}\right\} \leq 0\right)$ are the spatial frequencies and $k=\omega \sqrt{\varepsilon_{0} \mu_{0}}$ is the wavenumber of free space.

The calculation domain (square of length $A$, as of Fig. 1) is therefore discretized by $N=2^{n}$ discrete points $\left(n \in \mathbb{N}^{*}\right)$. The spatial frequencies are then given by

$$
k_{x}=k_{y}=(-N / 2: N / 2) \times \pi / A .
$$

The constructed Fourier pair for the near field can be written

$$
\begin{aligned}
& \tilde{\boldsymbol{E}}=\mathcal{F}\{\boldsymbol{E}\}, \\
& \boldsymbol{E}=\mathcal{F}^{-1}\{\tilde{\boldsymbol{E}}\},
\end{aligned}
$$

where $\mathcal{F}$ and $\mathcal{F}^{-1}$ stand for the forward and inverse Fourier transform, respectively.

The inverse dyadic $\underline{\underline{\tilde{G}}}^{-1}$ is defined as

$$
\underline{\underline{\boldsymbol{G}}}^{-1}(z)=e^{+j k_{z} z} \hat{\boldsymbol{x}} \hat{\boldsymbol{x}}+e^{+j k_{z} z} \hat{\boldsymbol{y}} \hat{\boldsymbol{y}} .
$$

This operator, referred in the following as back-propagation (step 3), is restrained in the visible spectrum $\left(k_{x}^{2}+k_{y}^{2} \leq\right.$ $k^{2}$ ) to avoid extreme amplification of the invisible spectrum ( $\mathcal{R}\left\{e^{+j k_{z} z}\right\}>0$, with $\mathcal{R}$ the real part operator). It is easy to notice that only the tangential-to-the-aperture components are back-propagated.

\section{REFERENCES}

[1] A. Epstein and G. Eleftheriades, "Huygens metasurfaces via the equivalence principle: design and applications," J. Opt. Soc. Am. B, vol. 33, pp. A31-A50, Feb 2016.

[2] Y. Zhao and A. Alù, "Manipulating light polarization with ultrathin plasmonic metasurfaces," Phys. Rev. B, vol. 84, p. 205428, Nov. 2011.

[3] N. Yu and F. Capasso, "Flat optics with designer metasurfaces," Nat. Mater, vol. 13, pp. 139-150, Feb. 2014.

[4] Y. Rádi, C. Simovski, and S. Tretyakov, "Thin perfect absorbers for electromagnetic waves: Theory, design, and realizations," Phys. Rev. Applied, vol. 3, p. 037001, Mar. 2015.

[5] G. Zheng, H. Mühlenbernd, M. Kenney, G. Li, T. Zentgraf, and S. Zhang, "Metasurface holograms reaching $80 \%$ efficiency," Nature Nanotechnology, vol. 10, p. 308, Feb. 2015.

[6] G. Lipworth, N. Caira, S. Larouche, and D. Smith, "Phase and magnitude constrained metasurface holography at W-band frequencies," Opt. Express, OE, vol. 24, pp. 19372-19387, Aug. 2016.

[7] C. Pfeiffer and A. Grbic, "Metamaterial Huygens' surfaces: Tailoring wave fronts with reflectionless sheets," Phys. Rev. Lett., vol. 110, p. 197401, May 2013.

[8] A. Alù, M. Silveirinha, A. Salandrino, and N. Engheta, "Epsilon-nearzero metamaterials and electromagnetic sources: Tailoring the radiation phase pattern," Phys. Rev. B, vol. 75, p. 155410, Apr. 2007.

[9] B. H. Fong, J. S. Colburn, J. J. Ottusch, J. L. Visher, and D. F. Sievenpiper, "Scalar and tensor holographic artificial impedance surfaces," IEEE Trans. Antennas Propag., vol. 58, pp. 3212-3221, Oct. 2010.

[10] G. Minatti, F. Caminita, M. Casaletti, and S. Maci, "Spiral leaky-wave antennas based on modulated surface impedance," IEEE Transactions on Antennas and Propagation, vol. 59, pp. 4436-4444, Dec 2011.

[11] M. Casaletti, M. Smierzchalski, M. Ettorre, R. Sauleau, and N. Capet, "Polarized beams using scalar metasurfaces," IEEE Trans. Antennas Propag., vol. 64, no. 8, pp. 3391 - 3400, 2016.

[12] X. Wan, T. Y. Chen, Q. Zhang, J. Y. Yin, Z. Tao, L. Zhang, X. Q. Chen, Y. B. Li, and T. J. Cui, "Manipulations of dual beams with dual polarizations by full-tensor metasurfaces," Advanced Optical Materials, vol. 4, no. 10, pp. 1567-1572, 2016.

[13] G. Minatti, F. Caminita, E. Martini, M. Sabbadini, and S. Maci, "Synthesis of modulated-metasurface antennas with amplitude, phase, and polarization control," IEEE Trans. Antennas Propag., vol. 64, pp. 39073919, Sept. 2016.

[14] M. Teniou, H. Roussel, N. Capet, G. Piau, and M. Casaletti, "Implementation of radiating aperture field distribution using tensorial metasurfaces," IEEE Trans. Antennas Propag., vol. 65, no. 11, pp. 58955907, 2017.

[15] O. Yurduseven, D. L. Marks, J. N. Gollub, and D. R. Smith, "Design and analysis of a reconfigurable holographic metasurface aperture for dynamic focusing in the Fresnel zone," IEEE Access, vol. 5, pp. 15055$15065,2017$.

[16] T. Isernia, P. Di Iorio, and F. Soldovieri, "An effective approach for the optimal focusing of array fields subject to arbitrary upper bounds," IEEE Trans. Antennas Propag., vol. 48, pp. 1837-1847, Dec. 2000.

[17] D. Iero, L. Crocco, and T. Isernia, "Constrained power focusing of vector fields: an innovative globally optimal strategy," Journal Electromagnetic Waves Applications, vol. 29, pp. 1708-1719, Sept. 2015.

[18] R. Piestun and J. Shamir, "Synthesis of three-dimensional light fields and applications," Proc. IEEE, vol. 90, pp. 222-244, Feb. 2002.

[19] M. Teniou, H. Roussel, M. Serhir, N. Capet, P. G., and M. Casaletti, "Tensorial metasurface antennas radiating polarized beams based on aperture field implementation," Int. J. Microwave Wireless Technol., vol. 10(2), pp. 161-168, 2018

[20] M. Teniou, H. Roussel, M. Serhir, N. Capet, G. Piau, and M. Casaletti, "Experimental validation of tensorial metasurfaces for the implementation of radiating aperture field distributions," IEEE Transactions on Antennas and Propagation, pp. 1-1, 2019.

[21] T. Hansen and A. Yaghjian, "Probe correction in the frequency domain," in Plane-Wave Theory of Time-Domain Fields: Near-Field Scanning Applications, pp. 253-269, Wiley-IEEE Press, 1999.

[22] S. Gregson, J. McCormick, and C. Parini, Principles of Planar NearField Antenna Measurements. IET, 2007.

[23] C. Balanis, Antenna Theory: Analysis and Design, 3rd Ed. Hoboken, NJ, USA: Wiley Interscience, 2005. 